\title{
ESTIMATION OF RELATIVE DNA DENSITY IN HETEROCHROMATIN FROM C-BAND KARYOTYPES (CRITICAL REMARKS)
}

\author{
W. TIMISCHL* AND J. GREILHUBER $\dagger$ \\ * Institut für Algebra und Diskrete Mathematik, Technische Universität Wien, \\ Argentinierstrasse 8, A-1040 Vienna \\ † Institut für Botanik, Universität Wien, Rennweg 14, A-1030 Vienna
}

Received 15.iv.83

\section{SUMMARY}

The paper deals with problem of estimating the ratio of DNA densities in heterochromatin and euchromatin from C-band karyotypes of related species, when the observations are subject to errors of measurement. Using a set of data published for Lolium it is shown that under realistic assumptions about the error variances no significant difference of the densities in the two types of chromatin can be detected.

\section{INTRODUCTION}

In a recent investigation it was found by Thomas (1981) that in Lolium C-band heterochromatin has approximately seven times as much DNA per unit metaphase chromosome as euchromatin. This result was obtained from the data on 6 species listed in his table 1 by means of the simple regression model

$$
M=c_{h} H+c_{e} E+f_{m}
$$

with $M$ being the DNA mass (in pg) per nucleus, $H$ the length of C-band heterochromatin, $E$ the length of euchromatin (both in $\mu \mathrm{m}$ ) and $f_{m}$ the error component of $M$ (thus it is assumed that both $H$ and $E$ are measured without errors). The least-squares estimates of the parameters $c_{h}$ and $c_{e}$ were found to be $\hat{c}_{h}=0.388$ and $\hat{c}_{e}=0.0537$. These estimates were interpreted as "average concentrations" of DNA in the two types of chromatin and it was concluded from these figures that the ratio $c_{e}: c_{h}$ is as low as approximately 1:7. Unfortunately, no attempt was made to discuss the statistical significance of this ratio, either by the author or by other authors who seem to have accepted this result even if it is not supported by their own cytophotometric data (see Lukaszewski et al., 1982). It is very doubtful if the value found for the ratio $c_{e}: c_{h}$ has any practical meaning at all because of the assumption that $H$ and $E$ can be observed without errors. We think that this assumption is not justified and it is our aim to show what happens if errors of measurement are taken into consideration. Two possible approaches for handling the problem are outlined in the next section; in model $A$ a linear relationship between mathematical variables is assumed, in model $B$ a structural relationship between random variables. 


\section{MODELS AND DISCUSSION}

\section{Model A}

It is first assumed that the variables $X_{1}=H, X_{2}=E$ and $X_{3}=M$ are each subject to an error of measurement, i.e.,

$$
X_{i}=X_{i}^{\prime}+f_{i} \quad(i=1,2,3)
$$

where the $X_{i}^{\prime}$ are the "true" variables which can not be observed because of some error random variables $f_{i}$. The $X_{i}^{\prime}$ are considered as non-random, i.e., as mathematical variables, which are linearly related according to

$$
X_{3}^{\prime}=a_{1} X_{1}^{\prime}+a_{2} X_{2}^{\prime} \text {. }
$$

Inserting (ii) into (iii) gives $X_{3}=a_{1} X_{1}+a_{2} X_{2}+f$ (where $f=f_{3}-a_{1} f_{1}-a_{2} f_{2}$ ), which is different from (i) because $X_{1}$ as well as $X_{2}$ are correlated with $f$ Thus this equation can not be handled as a simple regression problem unless both $\sigma_{f_{1}}^{2}$ and $\sigma_{f_{2}}^{2}$ are zero (see e.g., Kendall and Stuart 1979, p. 401). What remains is to construct a confidence region for the parameters $a_{1}$ and $a_{2}$ which are the concentrations of the two types of chromatin. In addition to (ii) and (iii) the error variables $f_{i}$ are supposed to be uncorrelated and each distributed as $N\left(0, \sigma_{f_{i}}^{2}\right)$. Let $\left(X_{1 j}, X_{2 j}, X_{3 j}\right)$ be the triple of variables corresponding to observation $j(j=1,2, \ldots, n)$. Then, according to Brown (1957, see also Brown et al., 1958) the sum of squares of perpendiculars from the points $\left(X_{1 j} / \sigma_{f_{1}}, X_{2 j} / \sigma_{f_{2}}, X_{3 j} / \sigma_{f_{3}}\right)$ on to the hyperplane (iii), i.e.,

$$
D=\sum_{j=1}^{n}\left(a_{1} X_{1 j}+a_{2} X_{2 j}-X_{3 j}\right)^{2} /\left(\sigma_{f_{1}}^{2} a_{1}^{2}+\sigma_{f_{2}}^{2} a_{2}^{2}+\sigma_{f_{3}}^{2}\right)
$$

has a $\chi_{n}^{2}$-distribution, and thus with $c$ satisfying $P\left(\chi_{n}^{2}>c\right)=1-\gamma$ it follows that $D \leqq c$ is a $100(1-\gamma)$ per cent confidence region for $\left(a_{1}, a_{2}\right)$. The equation $D=c$ defines a conic in the $\left(a_{1}, a_{2}\right)$-plane, which is-neglecting degenerate cases-a hyperbola if

$$
\Delta=\left(\sum_{j} X_{1 j}^{2}-c \sigma_{f_{1}}^{2}\right)\left(\sum_{j} X_{2 j}^{2}-c \sigma_{f_{2}}^{2}\right)-\left(\sum_{j} X_{1 j} X_{2 j}\right)^{2}
$$

is less than zero and an ellipse for $\Delta>0$. Consequently the confidence region for $\left(a_{1}, a_{2}\right)$ is bounded for $\Delta>0$ and unbounded for $\Delta<0$. An illustration of the latter case is given by figure 1, which is based on the data in Thomas' paper and on realistic assumptions about the error variances. Figure 1 also shows the straight lines $a_{2} / a_{1}=1$ as well as $a_{2} / a_{1}=1 / 7$ (the latter corresponds to the ratio of concentrations found by Thomas) and it is seen that there is no reason why one should rely more on $a_{2} / a_{1}=1 / 7$ than on e.g., the ratio $a_{2} / a_{1}=1$ corresponding to equal concentrations.

For a detailed discussion we refer to the article by Brown (1958) and also to the comments in the book by Kendall and Stuart (1979).

\section{Model $B$}

We now assume that $X_{1}^{\prime}$ and $X_{2}^{\prime}$ are random variables from which the new variables $Y_{1}^{\prime}=X_{1}^{\prime} / X_{3}$ and $Y_{2}^{\prime}=X_{2}^{\prime} / X_{3}$ (being both of dimension length per mass) are derived. Instead of equation (iii) the relation

$$
E\left(Y_{2}^{\prime}\right)=b_{1} E\left(Y_{1}^{\prime}\right)+b_{0}
$$




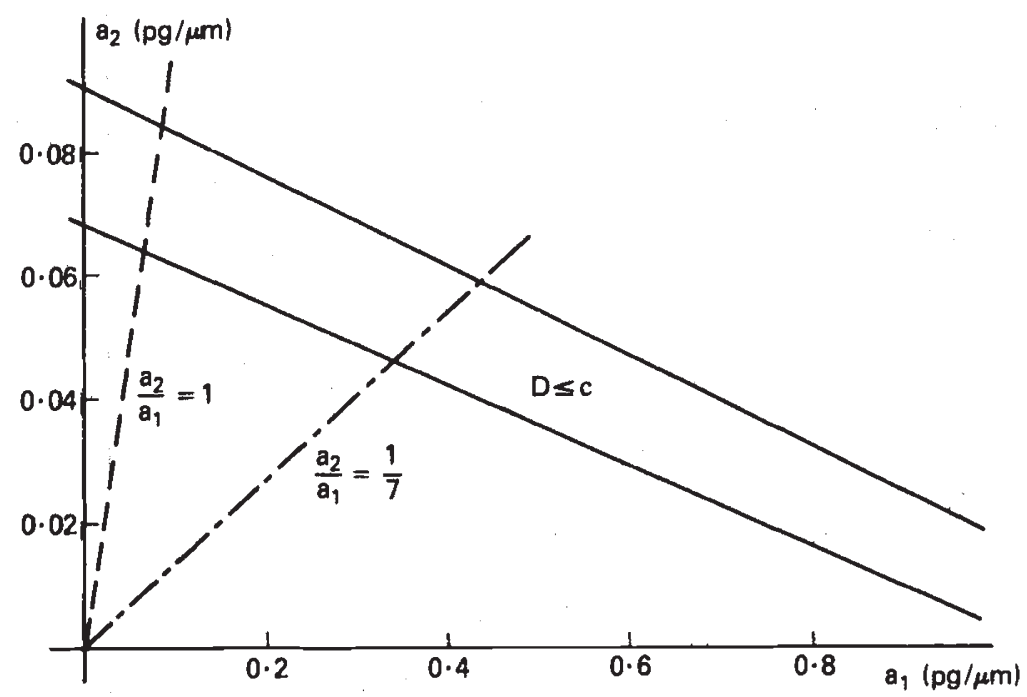

FIG. 1.95 per cent-confidence region for the parameters $a_{1}, a_{2}$ in equation (iii) based on the data of Thomas (1981, table 1 ). The standard deviations $\sigma_{f_{1}}$ and $\sigma_{f_{2}}$ were taken to be 10 per cent of the sample means of $X_{1}$ and $X_{2} ; \sigma_{f_{3}}$ was assumed to be 5 per cent of the corresponding sample mean.

is postulated between the expectations of $Y_{2}^{\prime}$ and $Y_{1}^{\prime}$, where $b_{1}$ is just the negative ratio of DNA concentration of $\mathrm{C}$-band heterochromatin and euchromatin and $b_{0}$ a constant. Model $B$ is thus based on a structural relationship between two (unobservable) random variables $Y_{2}^{\prime}$ and $Y_{1}^{\prime}$. What can be observed are the variables $Y_{i}=Y_{i}^{\prime}+g_{i}(i=1,2)$ where the error variables $g_{i}$ are again assumed to be uncorrelated and distributed as $N\left(0, \sigma_{g_{1}}^{2}\right)$ and the $Y_{i}^{Y_{i}}$ as $N\left(\mu_{Y_{i}}, \sigma_{Y_{i}}^{2}\right)$. If the ratio $\lambda=\sigma_{g_{2}}^{2} / \sigma_{g_{1}}^{2}$ of error variances is known, the $M L$-estimate $\hat{b}_{1}^{*}$ of $b_{1}^{*}=b_{1} / \sqrt{\lambda}$ is found to be (see e.g., Kendall and Stuart 1979)

$$
\hat{b}_{1}^{*}=\frac{1}{2 s_{Y_{1} Y_{2}^{*}}}\left[\left(s_{Y_{2}^{*}}^{2}-s_{Y_{1}}^{2}\right)+\sqrt{\left(s_{Y_{2}^{*}}^{2}-s_{Y_{1}}^{2}\right)^{2}+4 s_{Y_{1} Y_{2}^{*}}^{2}}\right]
$$

where $s_{Y_{1}}^{2}, s_{Y_{2}^{*}}^{2}$ are the sample variances of $Y_{1}$ and $Y_{2}^{*}=Y_{2} / \sqrt{\lambda}$ and $s_{Y_{1} Y^{*}}$ the sample covariance between $Y_{1}$ and $Y_{2}^{*}$. In order to test the hypothesis $b_{1}=b_{10}$ or to construct a confidence interval for $b_{1}$ the test statistic

$$
T=\sqrt{(n-2) \sin ^{2} 2\left(\beta_{1}^{*}-\beta_{10}^{*}\right) \frac{\left(s_{Y_{2}^{*}}^{2}-s_{Y_{1}}^{2}\right)^{2}+4 s_{Y_{1} Y_{2}^{*}}^{2}}{4\left(s_{Y_{1}}^{2} s_{Y_{2}^{*}}^{2}-s_{Y_{1} Y_{2}^{*}}^{2}\right)}}
$$

may be used which follows the $t$-distribution with $(n-2)$ degrees of freedom ( $n$ is the number of observations $\left(y_{1}, y_{2}\right)$ and $\beta_{1}^{*}=\arctan b_{1}^{*}, \beta_{10}^{*}=$ $\left.\arctan b_{10}^{*}\right)$. Assuming the error variances to be proportional to the squares of the corresponding means $\mu_{Y}$, we get from the data by Thomas $\lambda \approx 15$ and $\hat{b}_{1}=-6 \cdot 18$. From $(v)$ the 95 per cent-confidence interval $(-18 \cdot 3,1 \cdot 5)$ may be derived for $b_{1}$ which is rather large. It includes the $b_{1}$-value corresponding to the ratio $1 / 7$ of concentrations as well as the $b_{1}$-value corresponding to e.g., equal concentrations. 


\section{REFERENCES}

BROWN, R. L. 1957. Bivariate structural relation. Biometrica, 44, 84-96.

BROWN, R. L. AND FEREDAY, F. 1958. Multivariate linear structural relations. Biometrica, 45, 136-153

KENDALl, M. AND STUART, A. 1979. The advanced theory of statistics. Vol. 2. Griffin, London.

LUKASZEWSKI, A. J., WOZNA, J., APOLINARSKA, B. AND NAGANOWSKA, B. 1982. The DNA content of the individual chromosome of rye. Theor. Appl. Genet. 62, 145-148.

THOMAS, H. M. 1981. The Giemsa C-band karyotypes of six Lolium species. Heredity, 46, 263-267. 